\title{
Téoros
}

Revue de recherche en tourisme

\section{Accueil et formation : limites et possibilités}

\section{Sylvie Gagnon}

Volume 10, numéro 2, juillet 1991

Accueil et tourisme

URI : https://id.erudit.org/iderudit/1078959ar

DOI : https://doi.org/10.7202/1078959ar

Aller au sommaire du numéro

Éditeur(s)

Université du Québec à Montréal

ISSN

0712-8657 (imprimé)

1923-2705 (numérique)

Découvrir la revue

Citer cet article

Gagnon, S. (1991). Accueil et formation : limites et possibilités. Téoros, 10(2),

34-38. https://doi.org/10.7202/1078959ar d'utilisation que vous pouvez consulter en ligne.

https://apropos.erudit.org/fr/usagers/politique-dutilisation/ 


\section{Accueil et formation : limites et possibilités}

Cet article, loin de prétendre faire le tour de tout ce qui existe comme formation relative a l'accueil touristique au Québec, tente de cerner l'évolution de l'accueil chez-nous (avant la formation) et des enseignements dispensés sur cet aspect du tourisme dans les programmes réguliers de formation offerts dans nos institutions scolaires (principalement celui de Techniques touristiques), dans les Maisons du tourisme et les bureaux d'accueil aux préposés-es aux renseignements (comptoir et téléphonie) et, de plus en plus, dans les entreprises touristiques dans le cadre de programmes de formation sur mesure.

Ce tour d'horizon n'a pas pour but de présenter, en conclusion de notre article, le contenu du cours idéal à dispenser sur l'accueil mais bien plutôt de montrer, qu'audelà de l'enseignement des 1001 techniques d'accueil s ce qui est surtout garant de la qualité du travail effectué par les jeunes diplômés-es où les gens déjā en poste (dans une industrie saisonnière où la sécurité d'emploi est presqu'inexistante et les salaires guère alléchants) c'est l'importance qu'accordent les dirigeantses à leur personnel de contact et leur compréhension de l'approche systémique de gestion que nécessite un accueil de aqualité totalew.

C'est ce qui ressort de nos lectures mais surtout de nos fructueuses rencontres et discussions avec des professeurs-es, des responsables de programmes, des dirigeants-es du ministere du Tourisme et de bureaux de renseignements touristiques, des consultants et conseillers en formation et en main d'oeuvre(t) $\mathrm{et}$, bien entendu, avec des préposées à l'accueil. D'ailleurs nous tenons à les remercier chaleureusement pour le temps qu'ils-elles nous ont accordé et pour les documents qu'ils-elles ont mis à notre disposition.

\section{L'enseignement de I'Accueil au Québec avant la formation en tourisme}

Sans reprendre l'histoire et l'évolution de la formation en tourisme et du développement de cette industrie au Québec, il nous faut inévitablement effectuer un bref retour en arrière pour comprendre l'origine, les

Sylvie Gagnon est professeur au Departement de sciences administratives de I"UOAM objectifs et le contenu du premier cours à avoir porté le nom officiel de techniques $d^{\prime}$ accueil dans un des programmes de formation en tourisme (incluantl'hôtellerie et la restauration) reconnus par le ministère de l'Education. Ce même recul s'impose également pour saisir que ce n'est que tardivement qu'est apparu ce cours. En effet, bien que la formation dans ce domaine date principalement de la fin des années 1960 avec lacréation des CEGEPS et de l'Institut de tourisme et d'hôtellerie du Québec(2), ce n'est qu'en 1980 que l'on créa un cours portant précisémentsur l'accueil touristique. Pourtantl'hospitalitécet l'accueil, sous leurs différentes formes, préoccupaient déjă le Québec il y a plus de 100 ans comme en témoignent les quelques textes cités dans les lignes qui suivent.

\section{L'Accueil dans sa plus grande tradition européenne}

Dès la fin du XIXe siècle, nous raconte Henri-Paul Garceau dans son récent ouvrage sur l'histoire de l'hôtellerie au Québec ${ }^{3)}$, les règles de l'accueil et de l'hospitalité hôtelière, telles que développées dans les grands Palaces européens, étaient déjà transmises dans les premiers grands hôtels de Montréal et de Québec (le Windsor en 1878, le Queen's Hotel en 1892, le Château Frontenac en 1893) à une jeune main-d'oeuvre novice par des chefs et des maîtres d'hôtels européens fidèles aux deux grands maîtres Escoffier et Ritz:

Cette tradition s'exerçait manu militari par une discipline rigide. L'accueilse pratiquait selon une série de règles, dont le moindre manquement encourait de sévères remontrances ou parfois le renvoi. On se devait d'accorder aux clients une attention de tous les instants, d'etre à leur disposition pour les moindres désirs ou fantaisies, de leur manifester déférence et respect et leur témoigner, par une attitude courtoise et parfois obséquieuse, qu'ilsétaient très importants. ${ }^{(4)}$

\section{L'Accueil wbien de chez-nousw}

Parallèlement à ces spremières écoles de l'hospitalités se développaient également, en région, plusieurs entreprises familiales d'hébergement et de restauration qui, grâce à un accueil original et personnalisé dont les valeurs et le savoir-faire ont été transmis de génération en génération, attiraient elles aussi bon nombre de voyageurs qui en repartait satisfait. A preuve ces commentaires et anecdotes de l'époque rapportés par $\mathrm{H}_{+}-\mathrm{P}_{+}$Garceauls).

Mme Duberger, mère, est une femme de 70 ans. Elle ne se donne pas un instant de repos. Ses pensionnaires sont ses enfants. Il faut la voir à table, à côté de ses servants, les dirigeant, les stimulant. Sa voix domine toutes les autres. C'est un plaisir de la voir próvenir tous les désirs et tous les appétits. (Arthur Buies, 1884)

Au cours des 15 dernières années, nos hôtels se sont complètement métamorphosés. Ce ne sont plus des entreprises sans atmosphère et sans âme. Ce sont des centres d'hospitalité où vous êtes chez vous en entrant et d'où vous sortez avec deux amis de plus: le patron et la patronne. La nourriture? Excellente. Le service? Empressé. Le confort? Appréciable pour le prix demandé. (Paul Guévremont, comédien et directeur de tournées theatrales)

La bonne chère et le bon gîte ne se composent pas d'accessoires àquatre pattes comme le lit, la table et les chaises, mais de secrets et de recettes qui se transmettent de père en fils. Il est donc des dynasties qu'on respecte: celles des aubergistes et des cuisiniers.

[...]

La Pomme d'or est une des ces auberges à l'ancienne mode où l'on va faire un tour à la cuisine avant de se mettre à table. (Pierre Lefort, La Presse, 1933)

À la lecture de ces quelques témoignages, il nous est permis de penser que ces familles aubergistes ne sont pas étrangères à notre légendaire réputation - hélas, de plus en plus remise en question -de peuple hospitalier.

En 1940, cespetites entreprises touristiques comptaient 22783 employés et la grande hôtellerie 8300. Ainsi déjá plus de 30000 personnes, formées sur le tas, travaillaient à accueillir des gens au Québec ${ }^{(6)}$. 


\section{L'Accueil adevoir et obligation moralen et mise en garde contre I'Accueill trop intéressé}

D'autres organisations, en dehors des hôteliers et des restaurateurs, se préoccupaient aussi de 1'hospitalité et de l'accueil des touristes de plus en plus nombreux. Déjâ, en 1928 , G.-E. Marquis, fondateur de $1^{\prime}$ École des guides historiques de Québec, préfaçait un numéro du mensuel aL'école sociale et populaires ${ }^{(7)}$ consacré au tourisme où il citait des statistiques produites par la Banque de Montréal, le Bureau du tourisme de Montréal et le Club des automobiles de Québec demontrant la croissance de cette industrie pour justifier l'importance de ce numéro écrit par Eugène L'Heureux:

Il n'y a pas de doute qu'il y aura accroissement de touristes [...] et c'est pour que ces visiteurs y trouvent tout le confortet toutel'attention auxquels ils ont droitque M. Eugène L'Heureux, directeur du Progrès du Saguenay, a songé à ecrire une série d'articles sur le tourisme, tel qu'il le comprendet tel qu'il voudrait le voir accueillir par les habitants de la région du Saguenay. [...] nous croyons que les principes qui y sont développés conviennent également à toutes les localités de la Province ${ }^{(8)}$.

Dans son texte, empreint de morale et de patriotisme, Eugène L'Heureux donnent les conseils suivants (que nous nous sommes permis de résumer) à la population du Saguenay:

... que les hôtels et les maisons de pension offrent confort et propreté ainsi qu'une scuisine canadienne bien réussies faite par des kménagères averties\%:

... d'ajouter à notre idéal régional l'êlément artistique, le culte du beau ... afin d'embellir la région (entretien des propriétés, des maisons, des bâtiments de la ferme, etc.);

... de faire attention à son apparence et à sa tenue extérieure tout en se faisant un devoir de porter sune toilette bien françaisew;

... d'être poli et d'une «politesse obligeante au besoins tout en gardantsa fierté patriotique etsa dignité, d'être prévenant et soucieux du "fini en toutes choses $\%$;

.... de ne pas exploiter les touristes (par la fraude ou des prix exorbitants),

Sa conclusion ne laisse pas de doute quant à ses appréhensions d'un accueil trop intéressé: aÉducation, en vue de l'action et éducation en vue des réactions nécessaires, mais toujours education orientée vers la comprChension du devoir que nous avons tous d'envisager le côté moral en même temps que matériel des choses» ${ }^{(9)}$

\section{Le abonn Accueil et ses techniques de base.... universelles}

Un autre texte intitule Commentaires sur les 10 commandements du bon accueil et publié par la Chambre de commerce du district de Montréal, au début des années $1960^{(10)}$ dans le cadre d'une Semaine d'éducation touristique (l'ancêtre de la Semaine nationale de sensibilisation au tourisme?) fait également figure de précurseur dans le domaine de la formation en accueil. Cedocument de 8 pagess'adressait à la population montréalaise susceptible d'être en contact avec les touristes: chauffeurs de taxi, employés de grand magasin, préposés d'hôtel, etc. On y retrouve le pourquoi du sbon accueils (similaire au discours actuel) mais surtout des exemples de acomment faires pour observer les 10 commandements permettant de plaire aux touristes:

\section{Souriez \\ Saluez amicalement les visiteurs \\ Soyez courtois \\ Sachez dire merci! \\ Gardez votre bonne humeur \\ Exprimez de l'enthousiasme \\ Renseignez correctement le visiteur \\ Accordez une attention particulière ąux personnes âgées ou handicapées Évitez tout embarras au touriste Soyez un bon propagandiste de Mon- treal}

Il est suggéré, entre autre, de les rassurer, de leur faire sentir notre compréhension, notre bonheur de les aider, de bien amorcer la conversation ( $\ll$ Bonjour madame ou monsieur, puis-je vous êt(re utile? ?), d'avoir une bonne diction, d'être sincère, de demeurer poli même avec les insolents, de faire preuve de tact et de délicatesse, de les solliciter (quand c'est le cas) sans toutefois trop insister, de leur parler des derniers développements dans notre ville, des avantages qu'elle leur offre, de ne jamais laisser une de leurs questions en blanc, d'être aux faits des événements quotidiens, de connaître l'histoire de notre ville, sa géographie, etc. Bref, tout ce qu'il faut pour être la personne idéale que tout touriste souhaiterait rencontrer dès son arrivée... et tout ce que l'on tente encore d'enseigner aujourd'hui (30 ans plus tard) aux étudiant(e)s en tourisme, en hôtellerie et en restauration; aux préposés(es) à l'accueil du ministère du Tourisme, des Associations touristiques régionales (A.T.R.) et des offices municipaux; et au personnel de contact dans différentes entreprises touristiques désireuses d'améliorer la qualité de leur service.

\section{Les techniques d'accueil et la formation en tourisme et en hôtellerie}

Techniques touristiques : une formation en accueil de plus en plus ciblée sur les besoins du marchẹ du travail

\section{- La première définition du cours en} Techniques d' accueil et d' animation

C'est dans le cadre de ce programme régulier de formation de niveau collégial que s'est donné (et que se donne encore) le premier cours intitulé Techniquesd' accueil et $d$ animation comme nous $1^{\text {tavons men- }}$ tionné précédemment. Ajouté officiellement au programme lors d'une révision majeure de ce dernier en 1980, ce cours, dans la dernière édition (1989-1992) des Cahiers de l'enseignement collegial porte toujours la même définition:

\begin{abstract}
OBJECTIFS
Développer des attitudes et comportements exigés dans la profession de l'hôttesse et des préposés à l'accueil des visiteurs. Connaître les notions fondamentales et les qualités nécessaires à l'accueil et à l'animation des visiteurs étrangers.
\end{abstract}

\section{CONTENU}

Définir l'éthique professionnelle des gens de l'accueil. Savoir-vivre: l'art oratoire, la correspondance, art de la table et des vins, bienstance, etc. L'accueil et les relations publiques. Le milieu de travail: les différentes professions en tourisme et leur éthique. Technique de l'entrevue. Lecture des journaux. Communication: la langue et la diction. Les techniques audio-visuelles ${ }^{(11)}$.

Ce descriptif de cours, qui emprunte tantôt à un ancien cours du programme de 1975 portantsurl'éthique professionnelle (qui luimême empruntait déjà beaucoup à la formation en «service de table»), tantôt aux programmes en hôtellerie (art de la table et des vins, lecture des journaux), tantôt au secrétariat (pour ne nommer queceux-là) et qui s'adresse à la fois aux "hôtesses», aux préposés à l'accueil, aux «animateurs de visiteurs", n'est plus appliqué par aucun des professeurs-es des 5 collèges consultés.

\section{- La formation actuelle}

Afin de montrer l'évolution énorme qu'a subit ce cours depuis 1980 , grâce à des professeurs-es soucieux-ses de répondre aux besoins du marché du travaiil, nous présentons ci-après quelques exemples d'objectifs généraux et spécifiques extraits des plans de cours ${ }^{(12)}$ que nous avons consultés. Pour éviter les comparaisons inutiles (et non souhaitées par les profes- 
seurs-escontactés-es), c'est volontairement. que nous ne mentionnerons pas les sources de ces différents énoncés.

1. Quelques nouveaux objectifs gênéraux:

- Permettre aux élèves d'acquérir les valeurs, les attitudes et les comportements qui favorisent la qualité de l'accueil et de l'information des visiteurs.

- Faire prendre conscience aux participants de l'importance de l'accueil et de leur rôle éventuel dans une entreprise.

- Eveiller à la bonne façon de faire leur travail selon la mission particulière de l'entreprise.

- Motiver la personne à bien travailler.

2. Quelques nouveaux objectifs spécifiques:

- Etre capabled'analyser son comportement dans les situations d'accueil.

- Prendre conscience de son potentiel d'accueil.

- Connaître des moyens d'éviter les comportements agressifs au travail.

- Etre capable de gérer correctement les plaintes.

- Développer la capacité de communiquer adéquatement avec le visiteur, le client.

- Etre capable d'assurer son rồle de communicateur, de promoteur et d'informateur dans des situations diversifiées.

- Identifier les attitudes, les attentes et les besoins du visiteur, du client.

- Faire prendre conscience aux élèves de 1 'importance d'entretenir un environnement favorable à l'accueil.

Ces quelques exemples permettent d'abord de constater que la tâche est de plus en plus ciblée et rattachéc, en ce sens, à des fonctions de travail maintenant reconnues: accueillir, répondre à des questions, donner des informations, satisfaire des besoins. Dans certains cas on se limite mêtme clairement à la fonction de préposés-es à $1^{t}$ accueil dans un bureau d'information touristique. Il estaussi intéressant de constater qu"un des plans de cours aété développé selon une approche qui non seulement cible mieux la fonction de travail mais met continuellement celle-ci en rapport avec 1 'entreprise et sa mission.

Par rapport à cette tâche maintenant mieux identifiée, tous les plans de cours abordent le rôle et l'importance de l'accueil dans l'industrie touristique, les communications (verbales et non-verbales) et la psychologie (selon les types de visiteurs ou de clients: personnes âgées, personnes handicapées, etc., ou encore clientèle américaine, française, japonaise, etc.).
Un deuxième constat concerne l'aspect animation. Ce dernier faisant davantage référence à un groupe qu'à un individu, il semble être maintenant intégré au cours Guidage où forcément l'on fait référence à ce type de clientele. Une seule institution parmi celles consultées intègre encore l'animation et les groupes dans son cours sur l'accueil.

Un autre nouvel aspect présent dans la majorité des plans de cours concerne la connaissance de soi, de ses limites et de ses aptitudes afin d'évaluer ses forces et ses faiblesses par rapport aux exigences de l'accueil et du travail avec le public. Cet aspect n'est nullement abordé dans la définition officielle du cours (et mêne dans bon nombre de document consulté sur la formation en accueil) bien qu'il soit primordial à notre avis.

\section{Un nouveau programme qui accordera plus de temps à l'Accueil}

La réforme entreprise dans le milieu des années 1980 au niveau de la formation professionnelle au secondaire par le ministre Claude Ryan n'a pas manqué de toucher celle de niveau collégial, ce dernier étant à l'époque Également responsable du Conseil supérieur de l'éducation (de qui relève la Direction générale de l'enseignement collégial). La formation desagents de voyages (nouvelle orientation prise par les majorité des CEGEPS dans les années 1980 pour palier áu problème de placementdesélèves) se faisant depuis 1989 au niveau secondaire, la D.G.E.C. mis de l'avant une révision complète du programme de Techniques touristiques afin que celui-ci se donne de nouveaux objectifs lui permettant de se repositionner par rapport au nouveau programme pour les agents de voyages et par rapport également à la formation universitaire en tourisme donnée à l'Universitế du Québec à Montréal depuis plus de 10 ans.

De plus on imposa pour ce faire une nouvelle méthode d'élaboration de programme (utilisé systématiquement depuis 6 ans pour tous les programmes de formation professionnel de niveau secondaire) basée sur les tâches réelles effectuées en milieu de travail et sur les compétences à développer chez l'élève pour qu'il puisse les accomplir. Cette méthode, connue officieusement dans les milieux de formation sous le nom de améthode $\mathrm{X}{ }_{\text {, }}$ et cette révision en profondeur imposée par le gouvemement ne font pas encore l'unanimité au sein du Comité de coordination provincial du programme (composé d'un représentant de chacun des 6 collèges qui le dispense) mais, néanmoins, une version préliminaite est déjà sur la table et ce nouveau programme sera officiel sous peu.

Dans cette version préliminaire, les différentes compétences (synonyme de cours) que l'on développera chez l'élève et qui concernent les différentes facettes de l'accueil seront:

- Accueillir des clientèles touristiques et animer différents types de rencontre

- Caractériser les clientèles touristiques

90 hrs

S'exprimer en langue

seconde ou étrangère (I et II) 180 hrs

- S'exprimer en public $75 \mathrm{hrs}$

- Guider des visiteurs 75 hrs

- Accompagner des groupes 75 hrs

- Gérer un service d'accueil 75 hrs

Bien qu'il soit trop tôt encore pour se prononcer sur le contenu détaillé de chacun de ses modules, il va sans dire que l'enseignement de l'accueil et des connaissances nócessaires pour ce faire sera plus complet dans ce nouveau programme. Il nous faudra par contre attendre jusqu'en 1995 avant de porter un jugement sur la qualité de ces nouveaux-elles techniciensnes en tourisme.

\section{Hótellerie et restauration}

Bien que l'hôtellerie soit à l'origine de I'intérêt porté à l'accueil et à l'hospitalité chez-nous, on ne retrouve pas de cours qui porte spécifiquement sur l'accueil dans les programmes "Service de table» (secondaire) et *Techniques de gestion hôtelières (collégial) dispensés au Québec. Dans le cas du programme collégial (qui concerne davantage notre propos), l'accueil est principalement abordé dans les cours *Techniques de service de tablew (cours obligatoire) et «Réception d'hôtel» (cours de l'option «Hébergement»).

Bien que les bases de cette formation en hôtellerie et en restauration s' inspirent toujours de la plus haute tradition hôtelière européenne en ce qui concerne $l^{\text {'séthique et }}$ l'étiquette, il semblerait, d'après les propos de madame Janine Cornellier, professeure et conseillère pédagogique à l'I.T.H.Q. (depuis plus de 20 ans) qu'ici aussi l'importance de l'accueil et de la relation avec le client gagne du terrain. Comme c'est le cas d'ailleurs (nous le verrons plus loin) dans de nombreuses entreprises hôtelières et de restauration qui, de plus en plus, font appel à la formation sur mesure pour améliorer la qualité de l'accueil dans leur organisation.

\section{Éducation aux adultes et attestation d'études collégiales (A.E.C.)}

Outre les programmes réguliers mention= nés ici, il faut souligner que plusieurs CÉGEPS offrent, dans le cadre de la formation aux adultes, des cours en accueil et en animation touristiques. Parmi ceux-ci , apparaissent maintenantde plus en plus des 
cours destinếs à former ou recycler des préposés-es aux renseignements, desguides touristiques locaux et régionaux et du personneld'entreprises touristiques locales et régionales. Ces initiatives ponctuelles qui existent un peu partout en région dénotent une préoccupation certaine pour la qualité de l'accueil fait aux touristes par les différents intervenants du milieu.

\section{La formation sur mesure des préposés-es aux renseignements du ministère du Tourisme: quand $I^{i}$ Accueil devient Vente}

Bien que l'accueil concerne l'ensemble des intervenants qui sont en contact direct avec les visiteurs et les touristes, il est un groupe que 1'on associe davantage encore à cette fonction: ceux et celles qui constituent kla ligne de front $\%$, le premier $\alpha$ Bonjour $\%$, c'està-dire les préposés-es aux renseignements touristiques dans les bureaux gouvernementaux, régionaux et municipaux

Bien que nous $\mathrm{n}^{\dagger}$ ayons pu communiquer avec suffisamment de ces bureaux pour dresser un bilan de la situation actuelle en regard de la formation donnée à leur personnel, il nous est permis de croire que la situation s'est améliorée depuis le début des années $1970^{(13)}$ mais que dans plusieurs cas on n'accorde pas encore suffisamment d'importance à la qualité et à la formation des préposés-es qui y travaillent.

Nous nous limiterons donc ici à présenter dans ces grandes lignes le nouveau programme de formation des préposés-es mis de l'avant par le ministère du Tourisme en 1986 et mieux connu sous le nom de SPEX (le Service par excellence).

Avant, nous explique monsieur Renaud Plamondon, directeur du Service de l'accueil, le préposé aux renseignements avait une attitude passive: il répondait aux questions du touriste et lui remettait (ou lui envoyait par la poste), si nécessaire, une documentation thématique (carte routière du Québec, guide des hôtels, guide des campings, etc.). Avec la méthode SPEX, qui consiste en une technique d'entrevue «marketing», ce n'est plus le touriste qui mène l'entrevue mais plutốt le préposé.

Ce demier doit d'abord déceler ses besoins (en suivant des étapes bien précises), lui remettre la documentation pertinente et l'inciter «à consommer un produit supplémentaire ou complémentairè.

\section{Les objectifs du Ministère}

Ce programme de formation d'une durée de 10 jours doit être suivi par tous-tes les préposés-es aux renseignements. Il est dispensé par le personnel du Ministère à l'aide de documents pédagogiques conçus expressement à cette fin dont un «Cahier du formateurs ${ }^{(16)}$ et un aCahier du participants ${ }^{(15)}$ incluant des exercices et des mises en situation.

Ce programme vise bien sûr à développer les habilités nécessaires pour offrir un service de qualité mais surtout à homogénéiser ce service, qu'il soit offert au comptoir, au téléphone ou par correspondance.

La méthode SPEX qu'on yenseignedevrait permettre:

de sécuriser le préposé en structurant ses fonctions principales;

- à faciliter ses contacts avec le public:

- à l'initier à la dynamique des communications ${ }^{(16)}$.

En standardisant la façon de procéder à chaque aacte d'accueil devient également, pour les responsables, un outil de gestion efficace permettant d'évaluer et de contrôler, sur des bases communes, le rendement de chaque employé du réseau.

\section{Les 7 étapes du service par excellence}

Comme la majorité des cours sur l'Accueil, une partie de la formation donnée par le Ministère concerne l'importance de 1'industrie touristique au Québec et le rôle primordial que les préposés-es ont à jouer.

Une autre partie importante de la formation concerne le produit touristique: les régions, les publications existantes et les outils de travail thématiques mis a leur disposition ( $\alpha$ Baleines $*$, Croisieres-Grandes croisières\%, «Festivals et Événements», etc.).

Par contre, la nouveauté dans ce programme surmesure concerne la technique d'entrevue à utiliser pour donner le «service par excellences, service «qui dépasse les espérances du client» explique-t-on aux préposés-es. Cette technique, similaire au processus de vente, comporte 7 étapes que nous vous présentons ici brièvement.

\section{L'accueil}

Ici, on insiste sur l'importance du premier contactavec le visiteur, contact qui «fait en sorte que leclient se sente en confiance et le bienvenus. Il y est question du ton de la voix, de l'élocution, du sourire, de l'expression du visage et du corps et des premiers mots à prononcer.

\section{L'art d'être à l'écoute}

Dans cette deuxièméttape, on apprend aux participants et aux participantes les techniques de l'écoute active afin, dès le début de l'acte d'accueil, de acréer un bon climat de communication». Écouter c'est: aAvoir une attitude active; regarder celui qui parle; le laisser parler; être objectif; chercher la même longueur d'ondes; ... $x^{\text {(in }}$.

\section{La découverte}

La troisième étape du SPEX consiste à adécouvrir le réel besoin du touristew. Pour ce faire, le prếposé doit poser des questions: $\alpha D^{\prime}$ où? Quand? Pour combien de temps? Où? Pourquoi? Quel type d'hébergement? Comment? Avec qui? s ${ }^{(18)}$.

Ces nouvelles informations maintenant connues par le préposé permeturont à ce dernier de mieux cerner ce dont son interlocuteur a besoin. Ainsi, le visiteur qui, à priori, demande de 1'information sur la région de Charlevoix repartira avec des informations plus précises concernant «l'observation des baleiness, activité constituant son aréel besoin»

\section{Le résumé du besoin}

C'està cetteétape-ci que le préposés s'assure d'avoir bien cerné les besoins du touriste. Pour ce faire, il résume sa compréhension du besoin et tente d'obtenir l'accord de son interlocuteur. «Sij'aibien compris...; Donc vous désirez...; Ca va?; C'est bien cela que vous recherchez? ${ }^{(19)}$.

\section{La suggestion}

La suggestion est la réponse à la demande initiale du client une fois celle-ci précisée. Il s'agit donc pour le préposé d'identifier les différentes suggestions possibles etbien démontrer les avantages et les bénéfices au touriste: *Vous devriez faire des réservations le plus tôt possible car c'est le festival de Jazz à cette période à Montréal ${ }^{(0)}$.

\section{Le soutien}

Cette avant-dernière étape consiste à remettre la documentation pertinente et en expliquer le contenuet le moded'utilisation. Le choix de cette documentation sera faite en fonction de $\alpha$ l'entrevues que le préposé a fait passer au touriste plutôt qu'en fonction seulement de sa première question comme c'était le cas auparavant.

Ainsi, ce qui jadis était la tâche principale du préposé (remettre des documents) devient, avec cette nouvelle approche, une étape de fin de processus déterminée par les 5 étapes précédentes.

\section{La promotion}

Voilà l'élément le plus novateur, le plus *marketing de la méthode SPEX: tous les préposés doivent, avant de conclure un «acte d'accueil», «inciter le touriste à consommerdavantage le produit québécois» ${ }^{(2)}$ en lui suggérant des produits complémen- 
taires (ex.: un autre attrait à proximité de celui qu'il a I'intention de visiter) ou des produits supplémentaires (ex.: unc autre région, en plus de celle où il se rend, possédant tout ce qu'il recherche).

Cette dernière étape devrait donc faire sejourner le touriste plus longtemps ou le faire revenir au Québec dans un voyage ultérieur.

Cette nouvelle méthode, régulièrement améliorée depuis son application en 1986 , n'est peut-être pas encore parfaite mais elle aassurément amélioré la qualité de l'accueil faite aux touristes. Ce demier ne repart plus d'une Maison du tourisme avec une carte routière et un guide d'hébergement mais plutôt avec des documents remis en fonction de ses goûts, de ses intérêts, du temps dont il dispose, etc, et avec, en tete, des idées de eproduits à consonmers. Il s'agit là d'un changement majeur dans la aphilosophie d'accueils du ministère du Tourisme dont plusieurs offices municipaux et bureaux régionaux devraients 'inspirer pour former leurs préposés-es à l'accueil.

\section{La formation en entreprise}

Outre la formation dans le cadre de programmes réguliers offerts par les institutions scolaires publiques et privés et celle dispensée par le ministere du Tourisme, il ne faudrait pas oublier de mentionner celle donnée en entreprise et qui concerne le domaine de l'accueil.

En effet, de plus en plus d'entreprises touristiques font appel à des consultants, à des conseillers en formation, à des formateurs reconnus et à la Commission de la Formation Professionnelle (C.F.P.) pour faire dispenser à leur personnel des cours sur 1'accueil.

Quand ce ne sont pas elles qui font la demande, ce sont certains offices municipaux (ou la S.A.R.T.M. à Montréal) ou certaines A.T.R. qui les sollicitent dans le but d'améliorer la qualité de $1^{7}$ accueil en général sur leur territoire respectif.

A titre d'exemples d'entreprises ou d'organismes ayant fait appel à ce genre de cours pour leur personnelet pour montrer la diversité de ces demiêres, mentionnons:

- les caissiers/caissières de $I^{\prime}$ A.M.A.R.C. (la Ronde); le Mont Sainte-Anne;

- Le Gray Rocks dans les Laurentides; les agents de douanes;

Loews le Concorde à Québec:

- quelques réputés restaurants de Québec:

- le Chăteau Frontenac:

- le personnel du Cap Tourmente (Parc Canada):

- compagnies de croisières sur le fleuve: les préposés-es à 1'entretien de l'Ile Notre-Dame à Montréal.

Le contenu de ces cours varie d'une entreprise à une autre selon les besoins et selon la perception qu"ont ces différents dirigeants-es de l'importance de l'accueil. Ainsi, il peut s'agir d'une simple introduction à l'industrie touristique et à l'importance de l"accueil comme il peut êtrequestion, dans un contenu plusélaboré, des particularites d'une entreprise et des techniques d'accueil nécessaires à ce milieu. Dans d'autrescas encore, il peut s'agir de séminaires plus specialises où l'on apprend à des gestionnaires que dans les entreprises touristiques, la qualité tolale passe avant tout par une bonne gestion de son personnel de contact, celui-ci étant le premierconcernésur la schaîne deproductions.

Lademande pour cette formation sur mesure augmente sans cesse depuis quelques années. De septembre 1990 à juin 1991, la C.F.P. de la grande région de Québec a rejoint plus de 250 personnes oeuvrant dans des PME touristiques, soit 3 fois plus que l'année précédente. La formation donnée à ce personnel portait sur les techniques d'accueil et la qualite des services.

\section{L'Accueil: plus qu'une simple question de formation}

Au terme de cet article, nous sommes en mesure d'affirmer que l'enseignement des techniques d'accueil au Québec, depuis 10 ans, s'est beaucoup amélioré et s'arrime de plus en plus aux réalités du marché du travail.

En effet, les a 1001 trucs\%, les a 10 commandementss et le ecode d'êthiques du parfait préposé, avec ici et là quelques variantes sur le plan qualitatif de la méthode pédagogique utilisee, donnent suffisamment de connaissances aux individus (qui ểvidemment aiment travailler avec le public - parfois on semble l'oublier! -) pour qu'ils sachent accueillir convenablement le touriste ou le visiteur qui se présente à eux.

Le problème, comme le mentionne Murielle Drolet-Douville, conseillère en formation et spécialiste en techniques d'accueil, se situe souvent aux niveaux des responsables et des dirigeants qui souvent ne comprennent pas que la qualité de l'accueil fait aux clients par le personnel de contact passe par une philosophie de gestion de l'accueil qui implique toute l'organisation et non seulement le service du personnel ou le service à la clientele.

Sourire, être avenant, aimable et courtois c'est bien beaumais si l'entreprisen'accorde pas toute l'importance qu'il faut à cette tâche, ne valorisepasson personnel et ne lui foumit pas le cadre et la culture organisationnelle nécessaire pour produire un acte d'accueil de qualité totale, ce dernier pourra toujours sourire mais en vain.

Si les institutions scolaires ont fait leur bout. de chemin pour améliorer la qualité de la main-d'oeuvre qu'elles forment, c'est maintenant au tour des gestionnaires touristiques et des institutions qui forment ceux de demain de faire en sorte que le Québec retrouve ses lettres de noblesse au chapitre de $1^{7}$ accueil et de l'hospitalité.

\section{Notes explicatives}

[1] Nous remercions plus particulierement André Bergeren du CEGEP de Granby; Denis Sirois du College LaSalle; Alain Turootte du CEGEP de Matane et responsable du comité de coordination provincial; André Lessard. Lauronne Girard et Janine Cornellier de I'I.T.H.Q.; Murielle Drolet Douville, consultante en formation at professeure au College Merici; Serge Germain, guide-come dien, consulta ntet formateur; Renaud Plamondon, directeur du Service de l'accueil au ministere du Tourisme: Monique Slmard directrice de la S.A.R.T.M.; Marie-André Délisie, consultante et journal ste et Michelle Drolet, oonseillèreen maind'oeuvre a la Commission de la formation professionnalle de Québec

(2) Cf l'article d'André Bergeron intitulé "L'évolution de la formation en tourisme au Quebec" dans Téoros, vol. 2, no. 1, fourier 1983.

(3) GARCEAU, Henri-Paul, Chronique de l'hospitalité hôtelière du Québec de 1880 a 1940, Les Publications du Quebec et les Editions Méridien, Montréal, 1990, 212 p.

(4) Ibid., p. 36

(5) Ibid, p. 137 et pp. 200-201

(6) lbid. p. 208 .

(7) L'HEUREUX, Eugene, "Le tourisme, pourbe de richesse, Comment l'organiser" dans L'úcole sociale populaire, no. 160, Montreal, 1928

(B) lbid, pp, 3-4.

(9) lbid, p. 21.

(10) Cetexte, non signé et non daté Ket qui provient du Centre d'études du tourisme' a probablement été rédigé entre 1960 et 1965 puisqu'on y fait allusion. a quelques reprises, al la venue prochaine de IExposition Universelle de 1967

(11) Gouvermement du Quebec, Cahiers do l'enseignement collogial 19a9-1992, cahier 4 cours d'Etat, tome II, Direction genérale de l'enseignement colltgid, 1999, p. 1170.

112) Ont été consultes ou discutes les plans de cours actuels des institutions suivantes: I"I,T,H, , le college LaSalle, le college Merici, le CEGEP de Granby et le CEGEP de Matane.

(13) Cf. M.T.C.P., L'accueil ot I'information tourlstique au Quebec, vol. 1,2 et 3 . Service de la recherche, 1972

(14) MINISTERE DU TOURISME, Programmo do formation du probposa aux renseignements touristiquas - Cahler duformatour, sdition 1991, Servico de l'accueil, 307 pager.

(15) MINISTERE DU TOURISME. Programme de formation du préposá aux rensoignements touristiques - Cahier du participant, édition 1901 . Service de l'accueil, 236 piges.

(16) Ibid, p. 163 .

(17) Cahier du formateur, p. 213.

(1B) Ibid., p. 219

(19) Ibid., 0,220

(20) Ibid, p. 221

121) Ibid, p. 223 